\title{
Premature Thymic Involution and Multiple Sclerosis
}

\section{David G. Haegert}

Professor, Department of Pathology, McGill University, Canada

*Corresponding author: David Haegert, Duff Building, Room B22, 3775 rue University Montreal QC, H3A 2B4, Canada, Tel: 514-398-7192; Fax: 514-398-3465; E-mail: david.haegert@muhc.mcgill.ca

Received date: Apr 07, 2014, Accepted date: May 23, 2014, Published date: May 30, 2014

Copyright: ( 2014 Haegert DG. This is an open-access article distributed under the terms of the Creative Commons Attribution License, which permits unrestricted use, distribution, and reproduction in any medium, provided the original author and source are credited.

\begin{abstract}
It is uncertain as to whether similar or different mechanisms contribute to different subtypes of multiple sclerosis (MS). A unifying concept proposes that patients with both relapsing-remitting MS (RRMS) and primary progressive MS (PPMS) have premature thymic involution, which alters peripheral T-cell homeostasis and leads to T-cell alterations that contribute to the pathogenesis of both MS subtypes.
\end{abstract}

Keywords: Multiple sclerosis; Thymic involution; T-cell receptor excision circles (TRECs)

\section{Commentary}

Multiple sclerosis is a complex, heterogeneous disorder of the CNS An unresolved issue is whether similar or different mechanisms contribute to the different MS subtypes. Most think that peripheral immune mechanisms contribute to attacks in RRMS but not in PPMS [1]. An imbalance between regulatory and effector arms of the immune system may initiate attacks in RRMS [2]. In PPMS, favoured hypotheses include a neurodegenerative process [3] or a neuroinflammatory process confined within the blood-brain barrier [4]. As outlined below, cumulative data from various investigators suggest an alternate, unifying concept, that premature thymic involution leads to a shift in peripheral T-cell homeostasis, which contributes to the pathogenesis of both RRMS and PPMS [5].

Homeostastic mechanisms maintain body systems in equilibrium in the face of change [6]. T-cell homeostasis balances thymic output of newly generated naive T-cells, delivery of death and survival signals, differentiation of naive T-cells into memory T-cells, and homeostatic proliferation. Homeostatic proliferation compensates for the progressive thymic involution and decreasing thymic output that occurs with age [7].

T-cell receptor excision circles (TRECs) are by-products of T-cell receptor gene rearrangement in the thymus and can be quantified as a surrogate measure of thymic output [8,9]. Several report reduced thymic output in adult-onset RRMS, based on findings of reduced Tcell TREC content [5]. Since TRECs are cytoplasmic DNA fragments that do not replicate during mitosis, both reduced thymic output and increased proliferation can reduce T-cell TREC content $[8,9]$. In a study of TRECs that excluded proliferation, we found that young patients with RRMS and PPMS have thymic export of naive CD4 Tcells/day that is reduced to a level similar to controls 30-40 years older [10]. Balint et al. [11] expands on these observations, by showing that patients with pediatric-onset MS have reduced numbers of naïve and regulatory CD4 T-cells (Tregs) but increased numbers of memory CD4 T-cells; the naïve to memory CD4 $\mathrm{T}$-cell ratios are similar to older controls. In comparisons with age-matched controls, Tregs from these patients had reduced suppressive capacity [11], which is also described in adult-onset MS $[12,13]$. The duration of MS was short in the pediatric-onset MS cohort but the shifts in both naïve CD4 T-cell and Treg numbers were similar to those in adult-onset MS. Since pediatric- and adult-onset MS is likely the same disease, one may conclude that premature thymic involution, not MS-associated chronic inflammation, causes the T-cell changes in both adult-onset and pediatric-onset MS [5,11].

The relevance of the T-cell changes to pathogenic processes in MS is of considerable interest. Two possibilities can be entertained and these are not mutually exclusive. Increased immune responses to both self and environmental antigens occur in pediatric-onset MS, and these responses are abnormally high even in very young patients [14]. Treg dysregulation could explain these non-specific T-cell responses [11], which may target the CNS in genetically susceptible individuals. Alternatively, premature thymic involution in MS may lead to increased homeostatic proliferation. Homeostatic proliferation induced by self-antigens inevitably expands autoreactive T-cells and thus has relevance to the initiation of autoimmune responses [15]. Recently, we calculated the potential contribution of proliferation to the naive CD4 T-cell pools in healthy controls $(\mathrm{n}=68)$ and in patients with RRMS $(n=68)$ and PPMS $(n=20)$. We used a newly reported method based on TREC quantification, which demonstrated that $90 \%$ of naive CD4 T-cells in healthy adults is the product of peripheral T-cell proliferation [16]. In our study (manuscript in preparation), homeostatic proliferation generated on average $~ 92 \%$ of naive CD4 Tcells in healthy controls, whereas the average magnitude of this proliferation was significantly greater in MS; 95\% in patients with RRMS and $\sim 99 \%$ in patients with PPMS. These findings in MS are reminiscent of what occurs in an experimental mouse model in which lymphopenia induces increased homeostatic proliferation, which in turn initiates autoimmune diabetes [17].

What does premature thymic involution actually represent and when does it occur in MS? The normal human thymus grows only during the first year of life. Involution of thymic functional elements, including thymocytes and epithelial cells, then occurs in several phases that are independent of puberty. Phase 1 occurs from $\sim$ age 1 to $\sim$ age 10 , during which the thymus involutes at a rate of $\sim 5 \% /$ year. Subsequently, involution occurs at a rate of $\sim 3 \%$ /year until middle age and then at less than 1\%/year until old age [18-20]. The cohort reported by Balint et al. [11] had a mean age of MS onset of 13.5 years 
Citation: Haegert DG (2014) Premature Thymic Involution and Multiple Sclerosis. J Neurol Neurophysiol 5: 207. doi:

(range 7-17 years). It seems likely that some process altered the thymuses of these patients either during phase 1 of normal thymic involution or shortly thereafter. In our comparisons of adult-onset MS with controls, daily thymic export of CD4 T-cells decreased exponentially with age in healthy controls, consistent with the effects of normal thymic involution; daily thymic export was about $2 \times 10^{8}$ CD4 T-cells per day at age 35 but had decreased to around $5 \times 10^{7} \mathrm{CD} 4$ T-cells per day by age 60 . In contrast, daily thymic export was not only low in the young patients with RRMS and PPMS (less than $10^{6} \mathrm{CD} 4 \mathrm{~T}$ cells/day at age 35) but thymic export remained at a constant and similar low level with increasing age [10]. In another study, RRMS patients had reduced but remarkable constant levels of CD4 T-cell TRECs with increasing age [21]. Together, these findings suggest that thymic function is independent of the normal phases of thymic involution in MS. The distribution of the thymic export data and the CD4 TREC data in MS suggest that functional changes in the thymus occur well before disease onset, probably in childhood or adolescence $[10,21]$. It is tempting to speculate in MS that some process in childhood/adolescence arrests normal thymic involution, alters Tregs, and reduces thymic functional volume so that thymic export is maintained at a low but constant level throughout life. Possibly, some unknown intrinsic alteration in the normal thymic developmental program induces this premature involution. Alternatively, some extrinsic factor may affect the thymus, e.g. Epstein-Barr virus (EBV), which is associated with pediatric-onset MS [22] and has the potential to bind to EBV receptors in the thymus [23]. If premature thymic involution occurs early in life and has relevance to the pathogenesis of both RRMS and PPMS, the question remains as to why MS begins in childhood in only a minority of patients.

\section{References}

1. Hafler DA, Slavik JM, Anderson DE, O'Connor KC, De Jager P, et al. (2005) Multiple sclerosis. Immunol Rev 204: 208-231.

2. Vélez de Mendizábal N, Carneiro J, Solé RV, Goñi J, Bragard J, et al. (2011) Modeling the effector - regulatory T cell cross-regulation reveals the intrinsic character of relapses in Multiple Sclerosis. BMC Syst Biol 5: 114.

3. Trapp BD, Nave KA (2008) Multiple sclerosis: an immune or neurodegenerative disorder? Annu Rev Neurosci 31: 247-269.

4. Bradl M, Lassmann H (2009) Progressive multiple sclerosis. Semin Immunopathol 31: 455-465.

5. Haegert DG (2011) Multiple sclerosis: a disorder of altered T-cell homeostasis. Mult Scler Int 2011: 461304.

6. Baccala R, Theofilopoulos AN (2005) The new paradigm of T-cell homeostatic proliferation-induced autoimmunity. Trends Immunol 26: 5-8.
7. Takada K, Jameson SC (2009) Naive T cell homeostasis: from awareness of space to a sense of place. Nat Rev Immunol 9: 823-832.

8. Douek DC, McFarland RD, Keiser PH, Gage EA, Massey JM, et al. (1998) Changes in thymic function with age and during the treatment of HIV infection. Nature 396: 690-695.

9. Hazenberg MD, Otto SA, Cohen Stuart JW, Verschuren MC, Borleffs JC, et al. (2000) Increased cell division but not thymic dysfunction rapidly affects the $\mathrm{T}$-cell receptor excision circle content of the naive $\mathrm{T}$ cell population in HIV-1 infection. Nat Med 6: 1036-1042.

10. Haegert DG, Hackenbroch JD, Duszczyszyn D, Fitz-Gerald L, Zastepa E, et al. (2011) Reduced thymic output and peripheral naïve CD4 T-cell alterations in primary progressive multiple sclerosis (PPMS). J Neuroimmunol 233: 233-239.

11. Balint B, Haas J, Schwarz A, Jarius S, Fürwentsches A, et al. (2013) T-cell homeostasis in pediatric multiple sclerosis: old cells in young patients. Neurology 81: 784-792.

12. Viglietta V, Baecher-Allan C, Weiner HL, Hafler DA (2004) Loss of functional suppression by $\mathrm{CD} 4+\mathrm{CD} 25+$ regulatory $\mathrm{T}$ cells in patients with multiple sclerosis. J Exp Med 199: 971-979.

13. Haas J, Fritzsching B, Trübswetter P, Korporal M, Milkova L, et al. (2007) Prevalence of newly generated naive regulatory $\mathrm{T}$ cells (Treg) is critical for Treg suppressive function and determines Treg dysfunction in multiple sclerosis. J Immunol 179: 1322-1330.

14. Banwell B, Bar-Or A, Cheung R, Kennedy J, Krupp LB, et al. (2008) Abnormal T-cell reactivities in childhood inflammatory demyelinating disease and type 1 diabetes. Ann Neurol 63: 98-111.

15. Kohler S, Thiel A (2009) Life after the thymus: CD31+ and CD31human naive CD4+ T-cell subsets. Blood 113: 769-774.

16. den Braber I, Mugwagwa T, Vrisekoop N, Westera L, Mögling R, et al. (2012) Maintenance of peripheral naive $\mathrm{T}$ cells is sustained by thymus output in mice but not humans. Immunity 36: 288-297.

17. Datta S, Sarvetnick N (2009) Lymphocyte proliferation in immunemediated diseases. Trends Immunol 30: 430-438.

18. Steinmann GG, Klaus B, Müller-Hermelink HK (1985) The involution of the ageing human thymic epithelium is independent of puberty. A morphometric study. Scand J Immunol 22: 563-575.

19. Steinmann GG (1986) Changes in the human thymus during aging. Curr Top Pathol 75: 43-88.

20. George AJ, Ritter MA (1996) Thymic involution with ageing: obsolescence or good housekeeping? Immunol Today 17: 267-272.

21. Hug A, Korporal M, Schröder I, Haas J, Glatz K, et al. (2003) Thymic export function and $\mathrm{T}$ cell homeostasis in patients with relapsing remitting multiple sclerosis. J Immunol 171: 432-437.

22. Alotaibi S, Kennedy J, Tellier R, Stephens D, Banwell B (2004) EpsteinBarr virus in pediatric multiple sclerosis. JAMA 291: 1875-1879.

23. Paterson RL, Kelleher CA, Streib JE, Amankonah TD, Xu JW, et al. (1995) Activation of human thymocytes after infection by EBV. J Immunol 154: 1440-1449. 